\title{
CAISMOV24, a new human low-grade serous ovarian carcinoma cell line
}

\author{
Rodrigo Fernandes da Silva', Daniela Maira Cardozo', Gisele Olinto Libanio Rodrigues ${ }^{2,3}$, \\ Caroline Natânia de Souza-Araújo ${ }^{1}$, Natacha Azussa Migita ${ }^{2,3}$, Liliana Aparecida Lucci de Angelo Andrade ${ }^{1}$, \\ Sophie Derchain ${ }^{1,4}$, José Andrés Yunes ${ }^{1,3}$ and Fernando Guimarães ${ }^{1,4^{*}}$ (i)
}

\begin{abstract}
Background: The spontaneous immortalization of primary malignant cells is frequently assigned to their genetic instability during in vitro culturing. In this study, the new epithelial ovarian cancer cell line CAISMOV24 was described and compared with its original low-grade serous ovarian carcinoma.

Methods: The in vitro culture was established with cells isolated from ascites of a 60-year-old female patient with recurrent ovarian cancer. The CAISMOV24 line was assessed for cell growth, production of soluble biomarkers, expression of surface molecules and screened for typical mutations found in serous ovarian carcinoma. Additionally, comparative genomic hybridization was employed to compare genomic alterations between the CAISMOV24 cell line and its primary malignant cells.

Results: CAISMOV24 has been in continuous culture for more than 30 months and more than 100 in vitro passages. The cell surface molecules EPCAM, PVR and CD73 are overexpressed on CAISMOV24 cells compared to the primary malignant cells. CAISMOV24 continues to produce CA125 and HE4 in vitro. Although the cell line had developed alongside the accumulation of genomic alterations (28 CNV in primary cells and 37 CNV in CAISMOV24), most of them were related to CNVs already present in primary malignant cells. CAISMOV24 cell line harbored KRAS mutation with wild type TP53, therefore it is characterized as low-grade serous carcinoma.

Conclusion: Our results corroborate with the idea that genomic alterations, depicted by CNVs, can be used for subtyping epithelial ovarian carcinomas. Additionally, CAISMOV24 cell line was characterized as a low-grade serous ovarian carcinoma, which still resembles its primary malignant cells.
\end{abstract}

Keywords: Ascites, Cell culture, Comparative genomic hybridization, KRAS

\section{Background}

Ovarian cancer is the most lethal gynecological cancer, causing most of women's death. Its lethality is a consequence of the lack of symptoms or biomarkers enabling the early diagnosis of disease, and the propensity of malignant cells to seed the peritoneal cavity. The epithelium accounts for $90 \%$ of ovarian cancers [1,2]. Epithelial ovarian cancer (EOC) comprises four major histotypes, which are further classified based on their growth and molecular characteristics in slowly developing or type I

\footnotetext{
* Correspondence: fernando@caism.unicamp.br

'Faculdade de Ciências Médicas, University of Campinas, Campinas, SP, Brazil

${ }^{4}$ Women's Hospital "Professor Doutor José Aristodemo Pinotti" - CAISM,

University of Campinas, Rua Alexander Fleming 101, Campinas, SP 13083-881, Brazil

Full list of author information is available at the end of the article
}

tumors, and more aggressive or type II tumors [3-5]. The serous histotype is responsible for almost $70 \%$ of epithelial ovarian cancers, which are additionally differentiated into low (type I) and high grades (type II). The most aggressive subtype of serous ovarian carcinoma accounts for twothirds of all ovarian cancer deaths, making them the most extensively studied ovarian carcinoma $[3,4,6]$.

Biomarkers and molecular characterization of the tumors can represent a more accurate biological classification to separate and treat these tumors, rather than their site of origin. As an example, cancer antigen 125 (CA125) and human epididymis protein 4 (HE4) have been detected in the serum of ovarian cancer patients. Together, they enhance the sensitivity and specificity for the diagnosis of the disease $[7,8]$. Additionally, the 
identification of molecular surface markers on malignant cells can both contribute to the diagnosis and a better comprehension of tumor-host interactions [9-11]. Recently, molecular characteristics of tumors have become the new standard classification for clinical pathology. In this concern, methods such as comparative genomic hybridization allow the detection of deletions and duplications of genomic segments, known as copy number variation $(\mathrm{CNV})$. This method has been used to evaluate differences between cancer histotypes and putative target driver genes in EOC $[12,13]$.

Cell lines from various tumor types have been developed for use as a tumor model for controlled laboratory studies. Usually, cell lines have the facility to be cultivated, stored and shared between different laboratories. However, cell lines are known for their susceptibility to genetic and metabolic alterations, which can alter their characteristics in relation to the primary malignant cells. Ideally, the more a cell line resembles the primary malignant cells, the more useful it will be for scientific research. In vitro cultures of primary malignant cells and established cell lines have been widely employed as experimental models for the understanding of ovarian cancer biology, the evaluation of new therapeutic approaches and to search for tumor markers [14-17]. Currently, Broad-Novartis Cancer Cell Line Encyclopedia (CCLE https://portals.broadinstitute.org/ccle) includes 52 cell lines of human ovarian cancer [18]. Jacob et al. [17], recently published a review including 104 human cell lines originating from ovarian cancers or human ovarian surface epithelium. However, there are a limited number of the already established ovarian carcinoma cell lines, which have been well characterized as in vitro models. Domcke et al. [19] evaluated the genomic profiles of 47 ovarian cell lines in comparison to ovarian tumor samples, and found that some of the cell lines most commonly used as experimental models do not resemble their cognate tumor profile. Thus, new, well-characterized cell lines that resemble the different histological and molecular subtypes of ovarian neoplasia are still needed, particularly for lowgrade serous ovarian carcinoma [3, 4]. The goal of this study was to report the establishment of a new human epithelial ovarian carcinoma cell line (CAISMOV24), describing its phenotypic and molecular characteristics, and comparing genomic alterations between the cell line and its primary malignant cells.

\section{Methods}

\section{Patient and ascites sampling}

The patient was a 60-year-old woman, originally subjected to exploratory laparotomy for the diagnosis of ovarian neoplasia, procedure that was carried out at "Hospital da Mulher Prof. Dr. José Aristodemo PinottiCentro de Atenção Integral à Saúde da Mulher", the Women's Hospital of the University of Campinas (Campinas, Brazil). A non-resectable stage IIIC ovarian neoplasia was found from which a biopsy of the tumor mass was collected. The anatomical pathological diagnosis was consistent with a low-grade serous adenocarcinoma of the ovary (Fig. 1). Subsequently, the patient was treated with chemotherapy followed by surgery for cytoreduction.

Relapse occurred 1 year later, when ascites fluid originally used to initiate the in vitro culture of EOC cells was collected by sterile aspiration at the time of a laparotomy, in May 2011. The serum level of CA125 at the time of ascites collection was $2216 \mathrm{U} / \mathrm{ml}$. The patient died in August 2012 with pulmonary metastasis. The study was approved by the Research Ethics Committee of Unicamp (897/2011) and was registered on the Brazilian National Health Council (CAAE: 0807.0.146.00011). Signed informed consent was obtained from the patient prior sampling of ascites and blood.

\section{Initiation of the cell culture and maintenance of the cell line}

The ascites fluid was taken to our cell culture laboratory and processed immediately after sampling. Ascites fluid was transferred to $50 \mathrm{ml}$ conical tubes, the total volume was doubled by the addition of balanced salt solution (PBS; Nutricell, Campinas, Brazil) and $25 \mathrm{IU} / \mathrm{ml}$ of heparin was added (Liquemine, Roche, São Paulo, SP, Brazil). The cell fraction was isolated by centrifugation and washed twice in PBS $(400 \mathrm{~g} / 8 \mathrm{~min} / \mathrm{room}$ temperature). The final cell pellet was suspended in

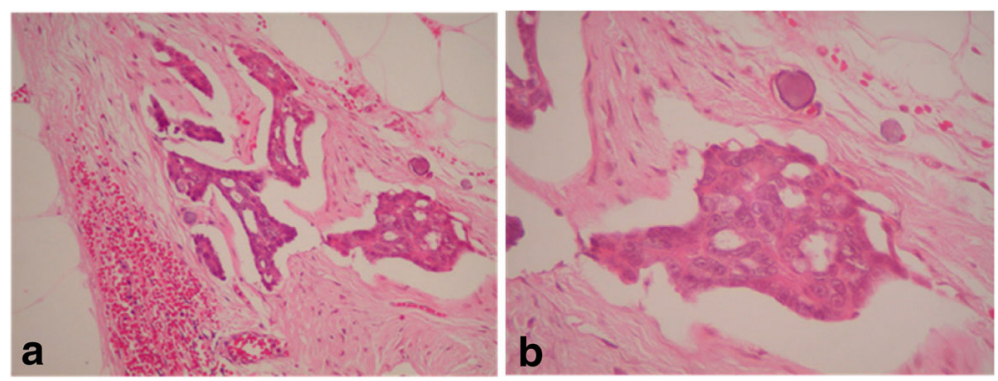

Fig. 1 Peritoneal implant of low-grade serous ovarian carcinoma. a obj 10x and b obj 40x 
HAM F10 medium supplemented with L-glutamine ( $2 \mathrm{mM}$, Nutricell, Campinas, Brazil) and fetal bovine serum (10\%, FBS; Nutricell, Campinas, Brazil). Cell number and viability were assessed using a hemocytometer and the trypan-blue exclusion method, respectively. Although the cell suspension was constituted by a diversity of cells at this time, the presence of tumor cells was noticed and the cell suspension was adjusted to $1 \times 10^{6}$ cells $/ \mathrm{ml}$ with culture medium. Thus, $10 \times 10^{4}$ cells were seeded in petri dishes (35 X $10 \mathrm{~mm}$; Corning, New York, USA), with supplemented culture medium added up to a volume of $2 \mathrm{ml}$ (resulting in a $5 \times 10^{4}$ cells $/ \mathrm{ml}$ suspension); dishes were placed in an incubator $\left(37^{\circ} \mathrm{C}, 5 \% \mathrm{CO}_{2}\right)$. The initial subcultures (the first 12 in vitro passages) were performed without a regular time period, ranging from 3 to 4 weeks, after which cells were detached with trypsin/EDTA (0.25\%; Nutricell, Campinas, Brazil), counted and seeded in new T25 flasks (JetBiofil, Guangzhou, China) at $10^{4}$ cells $/ \mathrm{cm}^{2}$ with supplemented HAM F10 medium. From the 12th in vitro passage onwards, the maintenance of the cultures and subculturing became regular. Thus, cultures were replenished with fresh medium every 2-3 days, and treated with trypsin/EDTA for cell re-plating every 2 weeks.

\section{Assessment of in vitro growth kinetics of the cells}

Quantification of the dividing capabilities of the cells was assessed several times during the 63rd and 100th in vitro passages. Thus, T25 culture flasks were seeded with $10^{4}$ cells $/ \mathrm{cm}^{2}$. Subsequently, replicates of the culture flasks were treated with trypsin/EDTA for cell detaching at different time points, and their number and viability were assessed using a hemocytometer and the trypan-blue exclusion method. The mean number of cells per centimeter square was expressed on a semi-logarithmic scale. Additionally, cell division in cultures was assessed by flow cytometry. For this, CAISMOV24 cells were labelled with violet proliferation dye 450 (VPD450, Becton, Dickinson and Company, Mountain View, USA) according to the manufacturer's instructions, prior to being seeded in T25 culture flasks at a density of $10^{4}$ cells $/ \mathrm{cm}^{2}$. Subsequently, cells were detached at different time points (days 3, 5, 7 and 10) for acquisition in a FACS Verse with FACS Suite software (Becton Dickinson, San Jose, USA). Stained cell suspensions were analyzed by setting the appropriate SSC/FSC gate on tumor cells and taking into account the fluorescence intensities on day 0 in labelled and unlabeled cells. The proliferation platform of FlowJo 7.1 software (Tree Star, Ashland, USA) was used for the data analysis.

\section{Immunophenotyping of cell line and biomarkers production in culture}

A flow cytometric-based assay was used according to standard procedures. Briefly, the cells were mixed with appropriate concentrations of fluorochrome-conjugated monoclonal antibodies (MAb), anti-CD326 (antiEpCAM, clone EBA), anti-HLA-class I (clone G46-2.6), anti-TGF $\beta 1$ (clone TW4-9E7), anti-CD39 (clone TU66), anti-CD73 (clone A2D) (Becton, Dickinson and Company, Mountain View, USA) and anti-CD155 (anti-PVR, clone 2H7) (eBioscience, San Diego, USA). Cells were incubated for $30 \mathrm{~min}$ in an ice bath and protected from light. After incubation, cells were washed twice with PBS and the final cell pellets were suspended for acquisition in a FACS Verse with FACS Suite software (Becton Dickinson, San Jose, USA). The human erythromyeloblastoid cell line $\mathrm{K} 562$ was used as a negative control for most of the surface molecules assessed in the assay. All samples were analyzed by setting the appropriate SSC/FSC gate on the tumor cells. FlowJo 10.0 software (Tree Star, Ashland, USA) was used for the data analysis.

The levels of soluble tumor markers CA125 and HE4 (human epididymis protein 4) were evaluated in the culture media taken between the 45th and 65th in vitro passages of the cells. An automatic chemiluminescencebased immunoassay was employed for this purpose. Thus, the CA125 II and Architect HE4 kits, combined with the Architect iSystem (Abott Diagnostics; Wiesbaden, Germany), were used according to the manufacturer's instructions to assess CA125 and HE4, respectively.

\section{Cytogenetic analysis}

The established cell line was subjected for routine karyotype and cytogenetic analysis. Briefly, cell cultures were treated with $100 \mu \mathrm{l}$ of KarioMAX colcemid solution $(10 \mu \mathrm{g} / \mathrm{ml}$; Life Techinologyes, Carlsbad, $\mathrm{CA}$, USA) and incubated for $5 \mathrm{~h}\left(37{ }^{\circ} \mathrm{C}, 5 \% \mathrm{CO}_{2}\right)$. Cells were detached with trypsin/EDTA (0.25\%), transferred to a $15 \mathrm{ml}$ conical tube and centrifuged (200 g/10 $\mathrm{min} /$ room temperature). The cells were subjected to hypotonic treatment with $0.075 \mathrm{M} \mathrm{KC1}$ for $8 \mathrm{~min}$ at $37{ }^{\circ} \mathrm{C}$, centrifuged and fixed at least 3 times for 10 min with a freshly prepared 3:1 mixture of methanol and acetic acid. Chromosome preparations were then made by dripping the cell suspension onto cold and wet clean slides and air-drying. The chromosome analysis was performed after staining of the slides for Giemsa G-banding. Twenty good quality metaphases were analyzed in order to describe the main findings, and digital images were obtained by using the BANDview System (Applied Spectral Imaging; Carlsbad, USA).

\section{Cell line authentication}

Analysis of short tandem repeats (STRs) was performed by Genomic Engenharia Molecular Ltda. (São Paulo, 
Brazil). STR analyses included markers for 21 loci on different chromosomes: D10S1237, D13S317, D16S539, D18S51, D19S433, D1S1656, D21S11, D22S1045, D2S1338, D3S1358, D5S818, D7S820, D8S1179, HUMCSF1PO, HUMFIBRA_FGA, HUMTH01, HUMTPOX, HUMVWA, Penta D, Penta E. STR profile of CAISMOV24 cell line was compared to that of other human cell lines, by using the tool available on line at http://www.dsmz.de/services/ser vices-human-and-animal-cell-lines/online-str-analysis.html. Cell identity is expressed as an evaluation value (EV) and calculated as $\mathrm{EV}=$ (Number of coincident peaks of STR profiles between cell lines A and B) X 2 / Total number of peaks of STR profiles in A and B. EV values greater than 0.9 indicate that the two cell types are derived from the same origin [20].

\section{Comparative genomic hybridization assay}

The Affymetrix Cytoscan HD platform microarray (Affymetrix, Santa Clara, USA) was employed to compare DNA alterations between the CAISMOV24 cells and their primary malignant cells. This array comparative genome hybridization platform contains 2.6 million markers for CNVs and 750,000 for single nucleotide polymorphisms (SNPs), enabling the high resolution detection of aberrations across the genome. Prior to DNA extraction, primary malignant cells were isolated from ascites by positive selection of the leukocytes, which was achieved by employing anti-CD45 MAb conjugated with beads and magnetic columns (MidiMacs separation system, Miltenyi, Bergisch Gladbach, Germany). Additionally, the cell suspension of the CAISMOV24 line was obtained by the detachment of cells from the established culture. DNA was obtained with an extraction kit based on columns with a silica membrane for DNA purification. (Illustra genomicPrep MiniSpin Kit, GE Healthcare, Buckinghamshire, UK). Finally, $250 \mathrm{ng}$ of genomic DNA from CAISMOV24 cells and their primary malignant cells was used for microarray hybridization using the Cytoscan HD platform (Affymetrix) according to the manufacturer's instructions. The Affymetrix CEL data files were converted into CYCHP files and the microarray data were analyzed using the Affymetrix Chromosome Analysis Suite 3.0 (ChAS 3.1) (Affymetrix). Analysis was performed using a setting of 50 consecutive probes and $100 \mathrm{kbp}$ in length for the detection of gains or losses. The intersection of genes present in genomic alteration regions, common between the primary malignant cells and CAISMOV24 cells, was analyzed using InteractVenn software (http://www.interac tivenn.net/). Relevant genes contained in the altered genomic sequences were compared with those reported by the Catalogue of Somatic Mutations in Cancer (COSMIC Welcome Trust Sanger Institute, http://cancer.sanger.ac.uk/).

\section{Mutation screening}

DNA and RNA were obtained from cryopreserved CAISMOV24 cells at 83rd in vitro passage with extraction kits based on columns with silica membrane and designed for DNA or RNA purification respectively (Illustra genomicPrep MiniSpin Kit, GE Healthcare, Buckinghamshire, UK). DNA and RNA were quantified with the Qubit dsDNA HS Assay Kit (ThermoFisher Scinteific, Waltham, USA) and RNA BR Assay Kit (ThermoFisher Scinteific) respectively. The quality of DNA extraction products was assessed through electrophoresis in agarose (1\%) stained with SYBR safe DNA Gel Stain (ThermoFisher Scinteific). RNA fragment length was assessed by a Bioanalyzer trace $\left(D_{200}\right.$ metric) using an RNA 6000 chip on an Agilent Bioanalyzer (Agilent Technologies Inc., Santa Clara, USA). DNA products were assessed for possible mutations in KRAS (exon 2), BRAF (exon 2) and TP53 (exon 2-11) genes by Sanger sequencing with the BigDye Terminator v3.1 Cycle Sequencing Kit (ThermoFisher Scientific) and capillary electrophoresis in an Applied Biosystems automated sequencer. Primers used for PCR are provided in Additional file 1: Table S1, and were based on the previously described by Arcila et al. [21]. Additionally, TruSight RNA Pan-Cancer Panel (Illumina, Inc., USA) was employed for transcriptome analysis of 1385 genes and 21,043 exons regions implicated in hotspot cancer pathways, following the manufacturer's protocol. Briefly, targeted RNA-seq libraries were prepared using $50 \mathrm{ng}$ of total RNA. The sample was subjected to RNA sequencing on an Illumina MiSeq (Illumina, Inc.) at 8 samples per flow cell $(\sim 3 \mathrm{M}$ reads per sample). Read mapping, gene expression information, variant calling, and fusion detection were performed using the RNA-Seq Alignment App with STAR aligner on BaseSpace Sequence Hub.

\section{Results \\ CAISMOV24 cell line establishment and in vitro growth kinetics}

Primary culture with cells from ascites was mainly composed of epithelial cells, and a small number of fibroblasts. However, the number of fibroblasts decreased until disappearing along with the initial in vitro passages. As previously mentioned, the first 9 to 12 initial subcultures were performed without a regular period of time (among 3 to 4 weeks), the period in which cell proliferation was slow and unable to cover the entire culture flask surface. After this period, cell proliferation became quicker and in vitro passages for the maintenance of cell culture became regular (every 2 weeks). To evaluate the reproducibility of the cell culture transformation from primary cells into the cell line, this procedure was repeated using cells from ascites which were maintained and 
cryopreserved. As a result, the same pattern of development was observed.

CAISMOV24 has been in continuous culture for more than 30 months and more than 100 in vitro passages. Figure 2a shows the typical epithelioid morphology of the established cell line. After cell plating, CAISMOV24 cells require $2-3$ days to exhibit their fully proliferation capability, when their average doubling population time was calculated to be $71.2 \mathrm{~h}$. Although the growth rate of the cell culture diminished from the 10th day, the cells continue to proliferate until covering the entire surface of the culture flask, reaching approximately 100,000 cells $/ \mathrm{cm}^{2}$ with $96 \%$ viability (Fig. 2b). VPD450-stained CAISMOV24 cells assessed by flow cytometry allowed the mean proliferation index to be calculated as $3.94 \pm 0.94$ times (Fig. 2c).

\section{Immunophenotyping and biomarker production in culture}

Figure 3 compares the expression of the HLA-class I, PVR (CD155), EpCAM (CD326), TGF $\beta 1, C D 39$ and CD73 surface molecules between CAISMOV24 cells and their primary malignant cells from ascites. The CAISMOV24 cell line was characterized by the expression of HLA-class I, EpCAM and PVR molecules. Additionally, CAISMOV24 cells express high levels of TGF $\beta 1$ and CD73, and low levels of CD39, molecules which are absent or modestly expressed on primary malignant cells. Serum biomarkers such as CA125 and more recently HE4 are important components in the treatment of women with adnexal masses. The production of serum biomarkers by CAISMOV24 cells in culture was confirmed by the detection of CA125 $(135.80 \mathrm{U} / \mathrm{ml}$ $\pm 56.26)$ and HE4 $(811.45 \mathrm{pmol} / \mathrm{ml} \pm 53.53)$ in the supernatant of CAISMOV24 cell cultures.

\section{Cytogenetic analysis}

The chromosomes of the cell line were initially analyzed by a routine karyotyping approach in the 45th in vitro passage. Figure 4 shows one representative G-banded karyotype depicting the main findings. According to standard nomenclature, the karyotype of this cell is denoted as 53 55, XX, +1 , add(1) (p36), add(2) (p25), +3, $\operatorname{add}(4)(\mathrm{q} 35),+5,+7,+8,-9,+\operatorname{dic}(9 ; ?)(\mathrm{p} 13 ; ?),+12,+14$, $+16,-19,+20,+22,-\mathrm{X},+\operatorname{mar}[\mathrm{cp} 51]$.

\section{Cell line authentication}

Tables 1 and 2 summarize the main findings regarding STR analysis. Comparisons of STR profiles between CAISMOV24 and other human cell lines did not match $\mathrm{EV}$ values greater than 0.9 , confirming the uniqueness of CAISMOV24 cell line.

\section{Comparative genomic hybridization assay}

CAISMOV24 cell line and its primary malignant cells were compared for chromosomal aberrations. For this purpose, a comparative genomic hybridization assay was

\section{a}
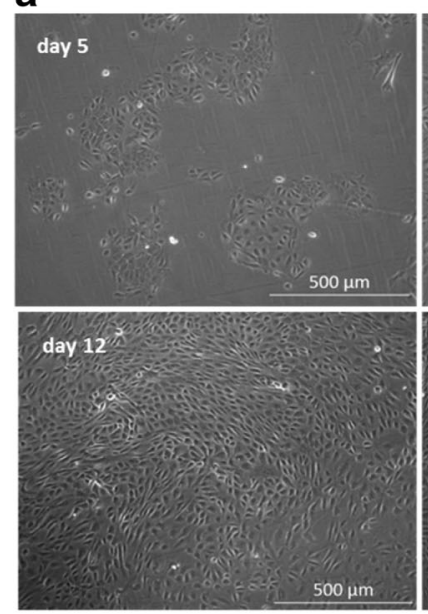
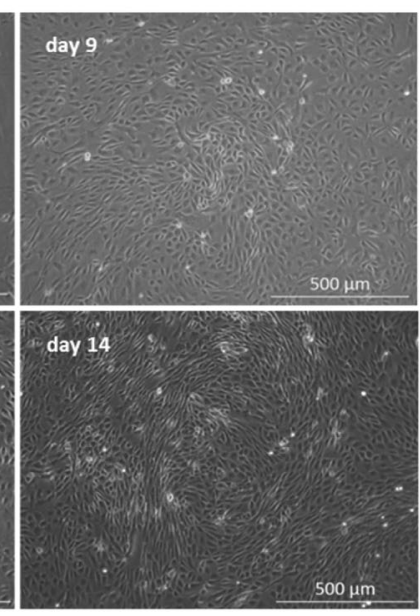

b CAISMOV24 proliferation profile
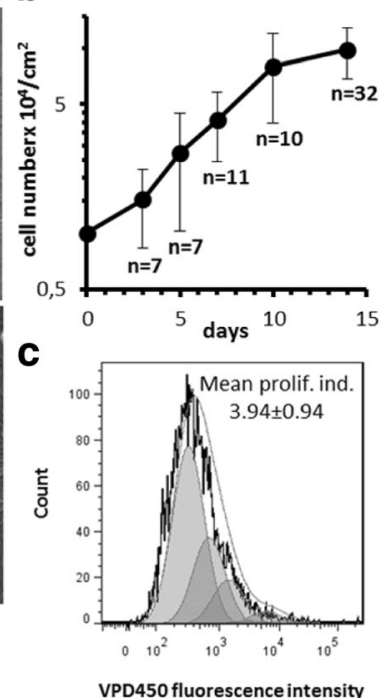

Fig. 2 a Different time points of the in vitro growth of the CAISMOV24 cell line. CAISMOV24 cells were launched at $10^{4}$ cells/cm ${ }^{2}$ in $\mathrm{HAM}_{\mathrm{F}} 10$ medium supplemented with $2 \mathrm{mM}$ L-glutamine and 10\% fetal bovine serum. b Representative growth curve for the CAISMOV24 cell line, assessed from the 63rd to the 100th in vitro passages. c Proliferation profile of CAISMOV24 cells assessed by flow cytometry on day 5, following cell labeling with violet proliferation dye 450 (VPD450); the shaded areas represent each of the new cell generations, which retained approximately half of the VPD450 fluorescence intensity of its parent cell. Mean proliferation index of CAISMOV24 cells resulted in $3.94 \pm 0.94$ times 


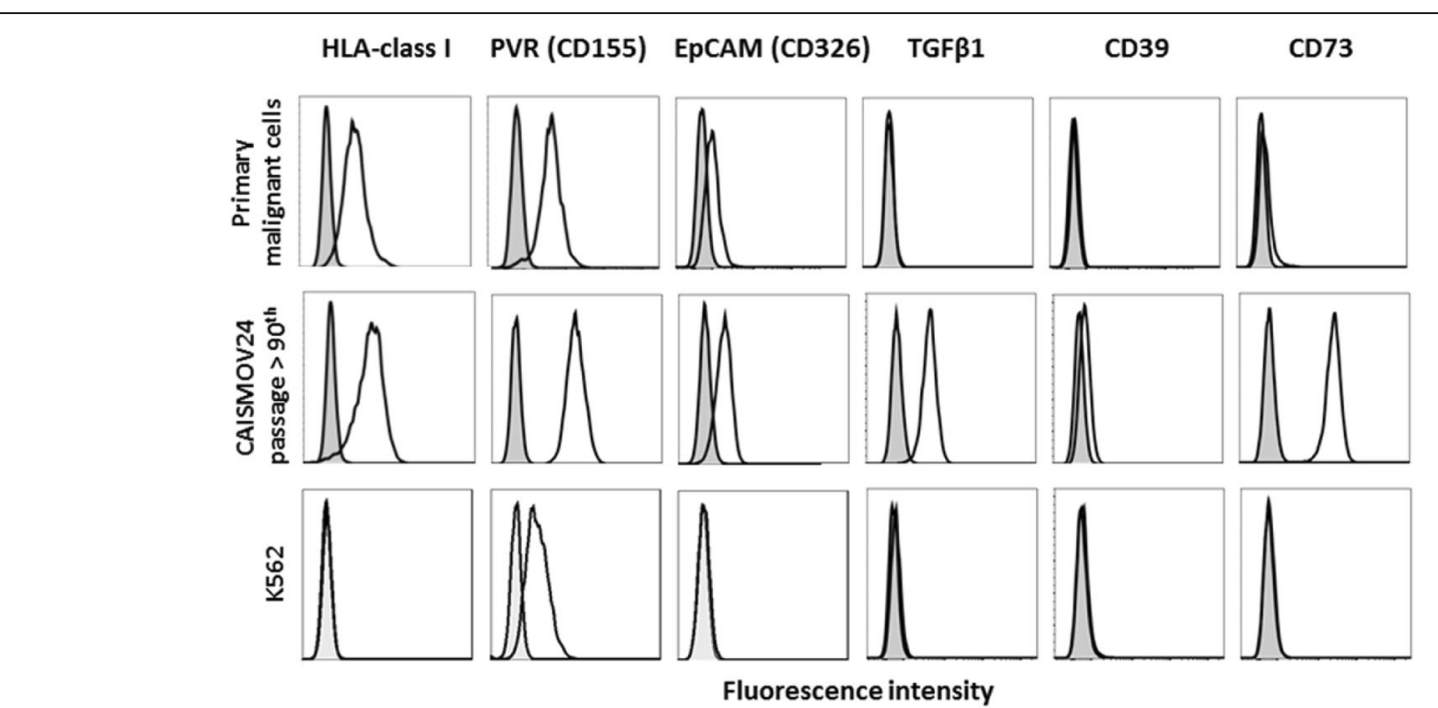

Fig. 3 Flow cytometric profiles representative of the fluorescence intensity of the molecules, HLA-class I, PVR (CD155), EpCAM (CD326), TGF- $\beta$ 1, CD39 and CD73, comparing the expression of these surface molecules between the CAISMOV24 cell line and its primary malignant cells. The cells were incubated with appropriate concentrations of fluorochrome-conjugated monoclonal antibodies. After incubation, cells were washed with PBS and the final cell pellets suspended for acquisition, using a FACS Verse flow cytometer. The K562 cell line was employed as a negative control for most of the surface molecules assessed in this assay

employed. Table 3 summarizes the main findings regarding CNVs. The total number of CNVs increased with the number of in vitro passages, with 28 detected in the primary malignant cells and 37 in the cell line assessed at the 83 rd in vitro passage. Chromosomes $4,10,15,17,18$ and 21 remained unaltered, both in the primary malignant cells and in the cell line. Additionally, in eight of the altered chromosomes, the number of CNVs remained the same for the primary malignant cells and the cell line. The most affected chromosomes

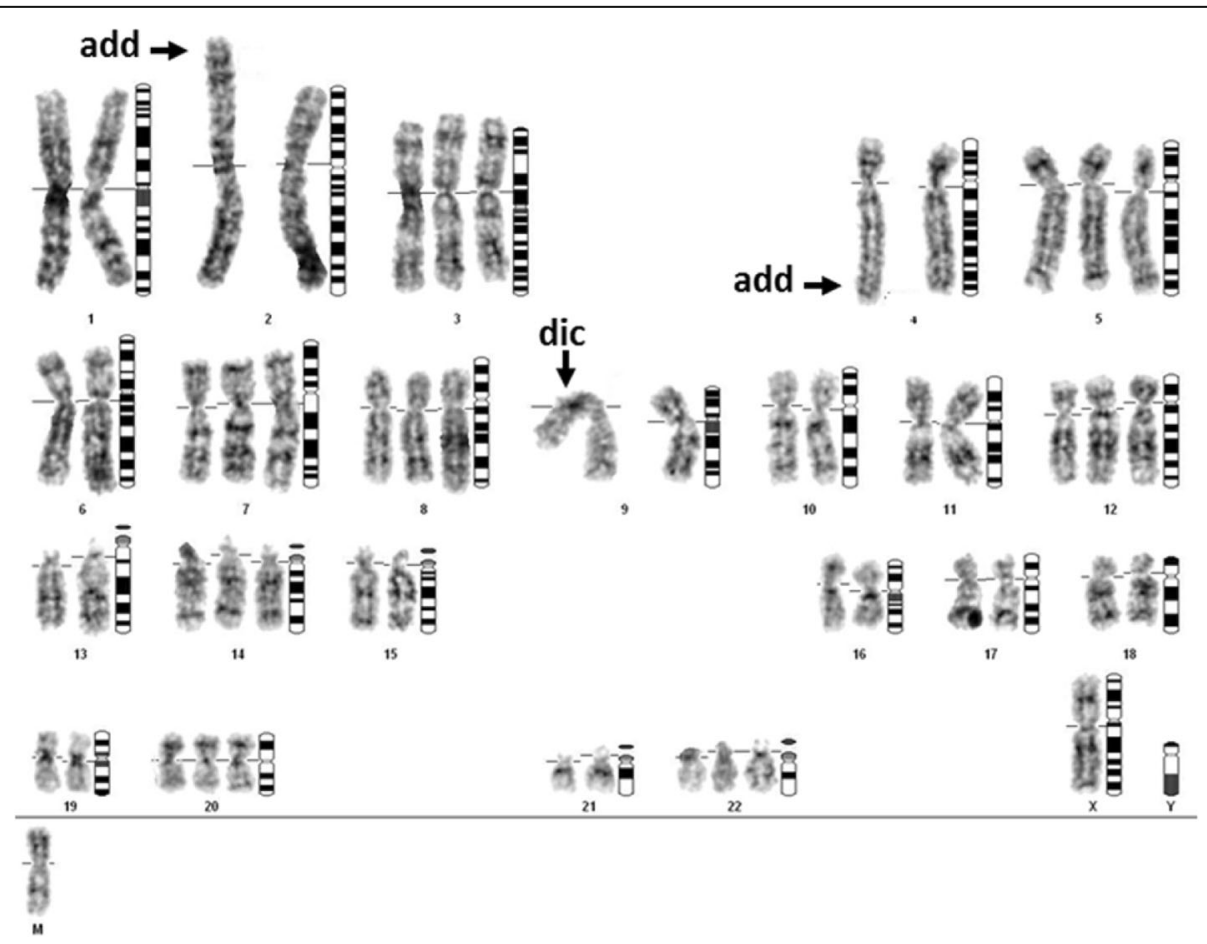

Fig. 4 Representative G-banded karyotype of a CAISMOV24 cell with 54 chromosomes. Dic = dicentric chromosome, add = additional material of unknown origin 
Table 1 Short tandem repeat (STR) analysis of CAISMOV24

\begin{tabular}{ll}
\hline Locus & Alleles \\
\hline D10S1237 & 21,19 \\
D13S317 & 12,11 \\
D16S539 & 12,12 \\
D18S51 & 19,12 \\
D19S433 & 14,13 \\
D1S1656 & $16,15.3$ \\
D21S11 & $32.2,30$ \\
D22S1045 & 16,16 \\
D2S1338 & 17,17 \\
D3S1358 & 17,16 \\
D5S818 & 13,12 \\
D7S820 & 12,8 \\
D8S1179 & 13,10 \\
HUMCSF1PO & 12,12 \\
HUMF13B & 10,9 \\
HUMFIBRA_FGA & 25,23 \\
HUMTH01 & 6,6 \\
HUMTPOX & 8,8 \\
HUMVWA & 17,17 \\
Penta D & 9,9 \\
Penta E & 13,7 \\
\hline
\end{tabular}

(with three or more CNVs) in the primary malignant cells were chromosomes $7,8,12,20$ and $\mathrm{X}$, which represented $60.7 \%$ (17 in 28 ) of the total CNVs. Chromosomes $1,7,8,9,12,16$ and 20 from the CAISMOV24 cell line had high numbers of CNVs (three or more CNVs), which represented $67.6 \%$ (25 in 37) of the total CNVs. Although CNVs were mostly represented by gains of genomic sequences $(86 \%$ of CNVs in primary malignant cells and $78 \%$ in CAISMOV24 cell line), CNVs also included losses of genomic sequences and hemizygous deletions of duplicated chromosome regions, characterizing copy neutral loss of heterozygosity $(\mathrm{cnLOH}$, genotyping provided in Additional file 2: Table S2, Additional file 3: Table S3 and Additional file 4: Table S4). Among losses of genomic sequences, there was one detected on chromosome 9 (ch9p21.3) of the primary malignant cells that was not detected in CAISMOV24 cell line. Extensive alterations, involving long genomic sequences, were detected in chromosomes $2,3,5,7,8,12,13,14,19$ and $\mathrm{X}$ (Fig. 5). The total size of genomic sequences contained in CNVs was higher in CAISMOV24 cells $(1,971,963 \mathrm{kbp})$ than in the primary malignant cells $(1,394,479 \mathrm{kbp})$.

The chromosomal regions affected by CNVs included 10,523 genes. Of these, 8798 genes were common for
Table 2 Short tandem repeat (STR) comparisons with DSMZ database

\begin{tabular}{|c|c|}
\hline EV & Cell No. Scored \\
\hline $1.00-0.95$ & 0 \\
\hline $0.95-0.90$ & 0 \\
\hline $0.90-0.85$ & 0 \\
\hline $0.85-0.80$ & 1 \\
\hline $0.80-0.75$ & 3 \\
\hline $0.75-0.70$ & 3 \\
\hline $0.70-0.65$ & 33 \\
\hline $0.65-0.60$ & 74 \\
\hline $0.60-0.55$ & 214 \\
\hline $0.55-0.50$ & 328 \\
\hline $0.50-0.45$ & 38 \\
\hline $0.45-0.40$ & 503 \\
\hline $0.40-0.35$ & 675 \\
\hline $0.35-0.30$ & 608 \\
\hline $0.30-0.25$ & 458 \\
\hline $0.25-0.20$ & 223 \\
\hline $0.20-0.15$ & 90 \\
\hline $0.15-0.10$ & 13 \\
\hline $0.10-0.05$ & 0 \\
\hline $0.05-0.00$ & 10 \\
\hline
\end{tabular}

STR profile of CAISMOV24 cell line was compared to that of other 3274 human cell lines available at DSMZ site, http://www.dsmz.de/services/ services-human-and-animal-cell-lines/online-str-analysis.html; Number of cell lines scored among different evaluation values (EV)

CNVs found either in the CAISMOV24 cell line or its primary malignant cell, 710 genes were present only in CNVs of the CAISMOV24 line, whilst 1015 genes were present only in the CNVs of the primary malignant cell (InteractVenn http://www.interactivenn.net/).

\section{Mutation screening}

Screening for typical mutations of serous ovarian carcinomas found that CAISMOV24 harbored KRAS mutation, together with wild type TP53, characterizing the cell line as low-grade serous ovarian carcinoma. Furthermore, transcriptome analysis confirmed the typical KRAS mutation (rs121913529; HGVS nomenclatures: NM_004985.4:c.35G > T, NP_004976.2:p.Gly12Val) frequently observed in lowgrade serous ovarian carcinomas. Transcriptome analysis also showed absence of gene fusions, and revealed several genes with SNP, in which its clinical relevance for the development of the serous ovarian carcinoma is unknown (Additional file 5: Table S5). Table 4 shows 15 variant genes without SNP location record and their frequency of alterations in CAISMOV24 cell line was higher than 0.35 . 


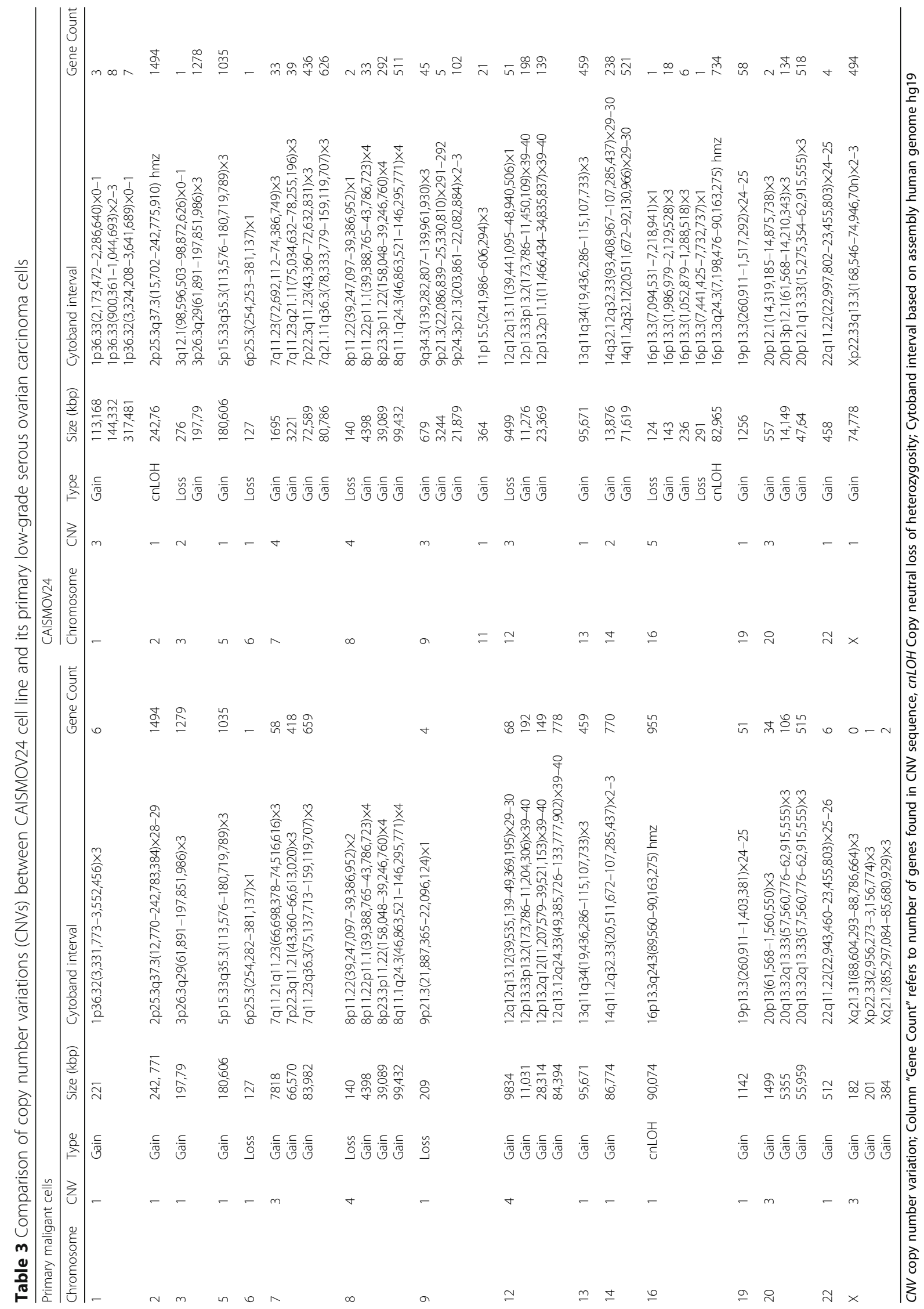



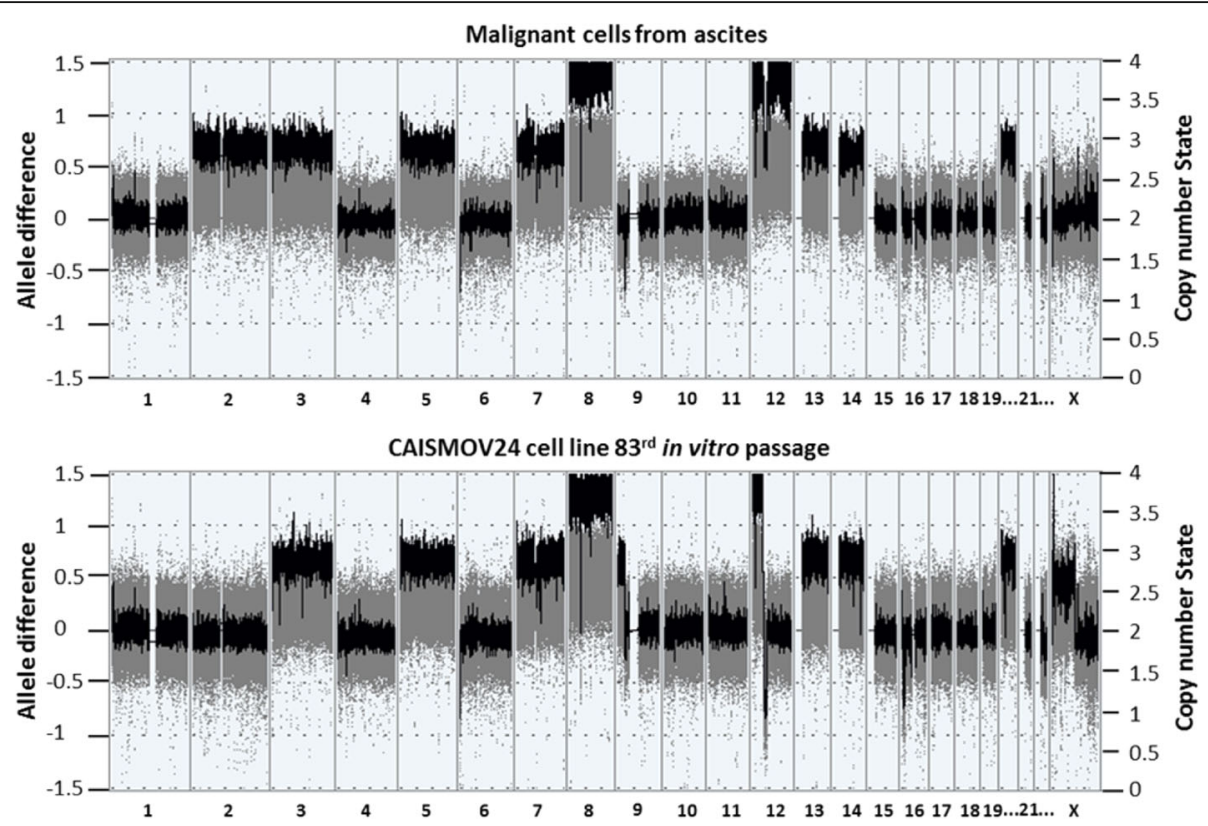

Fig. 5 Image captured from software ChAS (Affymetrix, USA) summarizing chromosomal aberrations found across the genome of the CAISMOV24 cell line compared with its primary malignant cells. Extensive alterations, involving long genomic sequences, were detected in chromosomes 3, 5 , $7,8,12,13,14,19$ and $X$, both in the CAISMOV24 cell line and its primary malignant cell. $X$ axis $=$ Chromosomes; Left $y$ axis $=$ Allele differences (gray); Right y axis = Copy number state (black)

\section{Discussion}

In this study a new low-grade serous ovarian carcinoma cell line, named as CAISMOV24, was characterized in terms of its in vitro cell growth, production of soluble biomarkers and expression of cell surface molecules. Additionally, CAISMOV24 was molecularly characterized and compared to its primary malignant cells for genomic alterations. In vitro models of well-characterized lowgrade serous ovarian cell lines are currently limited in the literature. CAISMOV24 resulted from the in vitro spontaneous immortalization of primary malignant cells from ascites that was associated with a low-grade serous ovarian carcinoma. Although malignant cells of ovarian neoplasia from either tumor tissue or ascites can be

Table 4 Gene mutations without SNP location record and with high alteration frequencies in CAISMOV24 cell line

\begin{tabular}{|c|c|c|c|c|c|c|c|}
\hline Gene & Chr & Position & Depth & Ref & Alt & Alt Freq & Variant Type \\
\hline AKAP9 & chr7 & $91,652,181$ & 18 & C & CAAC & 0.39 & inframe insertion \\
\hline HIST1H1D, HIST1H2APS3 & chr6 & $26,235,122$ & 186 & G & A & 0.39 & missense variant, downstream gene variant \\
\hline AKAP12 & chr6 & $151,674,121$ & 585 & A & AGGA & 0.41 & inframe insertion \\
\hline SPEN & chr1 & $16,202,753$ & 20 & G & A & 0.45 & missense variant \\
\hline NCOR2 & chr12 & $124,812,039$ & 117 & G & $\mathrm{T}$ & 0.47 & missense variant \\
\hline EP400 & chr12 & $132,554,157$ & 32 & G & A & 0.59 & missense variant \\
\hline ZNF687 & chr1 & $151,259,035$ & 5 & G & C & 0.60 & missense variant, upstream gene variant \\
\hline NOTCH1 & chr9 & $139,393,579$ & 38 & C & $\mathrm{T}$ & 0.63 & missense variant \\
\hline HMGA1, C6orf1 & chr6 & $34,211,292$ & 59 & A & AA & 0.78 & frameshift variant, feature elongation, downstream gene variant \\
\hline WHSC1L1 & chr8 & $38,148,080$ & 20 & C & $\mathrm{T}$ & 0.85 & missense variant \\
\hline KIF5B & chr10 & $32,311,775$ & 7 & C & G & 1.00 & splice donor variant \\
\hline PPP2R1B & chr11 & $111,631,542$ & 2 & C & $\mathrm{T}$ & 1.00 & splice donor variant \\
\hline RELN & chr7 & $103,230,181$ & 2 & $\mathrm{~T}$ & C & 1.00 & missense variant \\
\hline AKAP9 & chr7 & $91,652,181$ & 18 & C & CAAC & 0.39 & inframe insertion \\
\hline HIST1H1D, HIST1H2APS3 & chr6 & $26,235,122$ & 186 & G & A & 0.39 & missense variant, downstream gene variant \\
\hline
\end{tabular}


cultivated in vitro for a limited period, only a minority of the primary cell cultures may become cell lines [14]. Spontaneous immortalization of primary cultures of malignant cells is an event occurring at a very low frequency. As an example, O'Donnell et al. [11] reported the occurrence of only one spontaneous immortalization among 156 primary cultures generated from epithelial ovarian neoplasia, while all the other cultures entered senescence between the 2 nd and 8 th in vitro passages. The spontaneous immortalization of primary cultures is frequently assigned to the genetic instability of malignant cells and accumulation of genomic alterations, such as the gain or loss of genomic sequences, during in vitro culturing. However, this is a poorly understood and little documented phenomenon. Additionally, these genomic alterations can affect cell lines, driving them away from the cognate tumor profile and, thus, limiting their usefulness as experimental models.

Genomic alterations in low-grade serous ovarian carcinomas have been described as intermediate between borderline and high-grade serous ovarian carcinomas [22-24]. However, CNVs can be remarkably heterogeneous either in number or in their genomic location, among different low-grade tumors [25]. Although our results showed that CAISMOV24 cell line had arisen alongside the accumulation of genomic alterations, most of them were related to CNVs already present in the primary malignant cells. Additionally, some of these CNVs were reported to be shared by low-grade serous ovarian carcinomas. Thus, among the genomic alterations detected in the CAISMOV24 cell line and its primary malignant cells, copy number gains in chromosomes 8,12 and 20 were mentioned by Fernandez et al. [25] as commonly detected in low-grade serous carcinoma cell lines. Similarly, chromosome 4 was among those that did not harbor genomic alterations.

Our results showed that few CNVs were found exclusively in the cell line or in its primary malignant cells. Particularly, hemizygous deletion at ch9p21.3 was observed predominantly in primary malignant cells, but not in CAISMOV24 cells. This observation supported the low-grade origin of our primary cells, since hemizygous or homozygous deletions at ch9p21.3 have been reported as one of the most frequent copy number loss observed in low-grade serous ovarian carcinomas [22, 25]. However, the absence of this CNV in CAISMOV24 cells suggests that cell line could have emerged from specific cell clones of the primary malignant cells. Similarly, Lee et al. [26] showed that peritoneal metastasis arises from cellular clones with little accumulation of $\mathrm{CNV}$ in relation to its primary tumor in patients with high-grade serous ovarian cancer. Taken together, these observations suggest that similar mechanisms could be operating in vivo and in vitro systems. Thus, CAISMOV24 cell line shall have evolved from a process involving both, expansion of specific cell clones in addition to the enlargement or loss of altered pre-existing genomic sequences in primary malignant cells.

Although copy number alterations and gene mutations are caused by independent processes, it has been suggested that $\mathrm{CNV}$ profiles can be used for a better characterization among different histotypes of epithelial ovarian cancer [12], since they could harbor histotypespecific gene mutations. Additionally, CNVs could encode genes which drive growth [13, 27] and could alter gene expression or disrupt regulatory mechanisms leading to phenotypic alterations of the malignant cells in culture. Typically, low-grade serous ovarian carcinoma has few somatic mutations, which are mainly found in genes associated with the mitogen-activated protein kinase pathway such as KRAS, BRAF and NRAS [23, 25, 28]. KRAS and BRAF mutations are found in a proportion of low-grade serous carcinomas and, frequently, they are mutually exclusive. This is in contrast to high-grade serous carcinomas, which mutations include TP53 gene and rarely $K R A S[25,29]$. Accordingly, our results showed that CAISMOV24 cell line harbors KRAS mutation, which is relatively frequent in recurrent low-grade serous carcinomas [30]. The transcriptome analyses not only confirmed CAISMOV24 cell line as a low-grade serous carcinoma, but also revealed several genes with SNPs, which clinical relevance for development of serous ovarian carcinomas has being under evaluation. In this context, HMGA1 over-expression was observed in epithelial ovarian carcinomas [31], while NOTCH1 has been correlated with ovarian cancer development and a poor prognosis [32, 33].

In this study, immunophenotyping of the CAISMOV24 cell line was performed in an attempt to characterize the cells in relation to the expression of surface molecules, frequently observed in epithelial ovarian carcinomas, which have also been implicated in the failure of an immune response against tumors. Thus, the epithelial origin of CAISMOV24 cells was confirmed by the expression of EpCAM and PVR, two molecules expressed by ovarian epithelial cells, which are frequently overexpressed in ovarian carcinomas $[19,34,35]$. Additionally, expression of the HLA-class I molecule was not lost as a consequence of the malignant transformation of the ovarian epithelial cells $[36,37]$. Our results showed that CAISMOV24 cells express high levels of TGF- $\beta 1$ and CD73, and low levels of CD39, molecules that were absent or modestly expressed on the primary malignant cells. All of these molecules have been implicated in impaired immune response against ovarian carcinomas, either by direct TGF- $\beta 1$-mediated effects $[9,38,39]$, or by indirect effects resulting from the breakdown of ATP into adenosine by $\mathrm{CD} 73$, which promotes the 
immunosuppression of T cytotoxic and NK lymphocytes $[9,37]$. Serum biomarkers such as CA125 and more recently HE4 are important components in the treatment of women with adnexal masses. HE4 is a glycoprotein with low expression in normal ovarian tissue, higher in non-ovarian cancer tissue and highest in ovarian cancer tissue. HE4 as a single tumor marker has been reported to be as good as CA125 for the detection of ovarian cancer. HE4 and CA125 together enhance the sensitivity and specificity for the diagnosis of ovarian cancer $[7,8]$. In this study, the in vitro production of CA125 and HE4 by CAISMOV24 cells was confirmed, stressing the similarities between the cell line and its primary malignant cells.

\section{Conclusion}

In this study, the CAISMOV24 cell line was compared to its primary malignant cells. Our results corroborate with the idea that genomic alterations, depicted by CNVs, can be used for subtyping epithelial ovarian carcinomas. Additionally, CAISMOV24 cell line was characterized as a low-grade serous ovarian carcinoma, which still resembles its primary malignant cells.

\section{Additional files}

Additional file 1: Table S1. Primers. Primers used for PCR amplification prior to Sanger sequencing of KRAS, BRAF and TP53 genes. (DOCX $15 \mathrm{~kb}$ )

Additional file 2: Table S2. $L O H$ ch2p25.3 CAISMOV24. Genotyping of hemizygous deletions of duplicated chromosome regions (copy neutral loss of heterozygosity or $\mathrm{cnLOH}$ ) in chromosome 2 of CAISMOV24 cell line. (XLSX $5208 \mathrm{~kb}$ )

Additional file 3: Table S3. $L O H$ ch16p13.3 primary cells. Genotyping of hemizygous deletions of duplicated chromosome regions (copy neutral loss of heterozygosity or $\mathrm{cnLOH}$ ) in chromosome 16 of primary malignant cells. (XLSX $1738 \mathrm{~kb})$

Additional file 4: Table S4. $L O H$ ch16p13.3 CAISMOV24. Genotyping of hemizygous deletions of duplicated chromosome regions (copy neutral loss of heterozygosity or cnLOH) in chromosome 16 of CAISMOV24 cell line. (XLSX $1750 \mathrm{~kb})$

Additional file 5:Table S5. CAISMOV24 PanCancer transcriptome. Transcriptome data of CAISMOV24 cell line. (XLSX 531 kb)

\section{Abbreviations}

ATP: Adenosine triphosphate; CA125: Cancer antigen 125; CCLE: Cancer Cell Line Encyclopedia; CD: Cluster of differentiation; $\mathrm{CnLOH}$ : Copy neutral loss of heterozygosity; CNV: Copy number variation; COSMIC: Catalogue of Somatic Mutations in Cancer; DNAM-1: DNAX accessory molecule-1; EDTA: Ethylenediamine tetraacetic acid; EOC: Epithelial ovarian cancer; EpCAM: Epithelial cell adhesion molecule; FACS: Fluorescence-activated cell sorting; FBS: Fetal bovine serum; FSC: Forward scatter; HE4: Human epididymis protein 4; Mab: Monoclonal antibody; PVR: Poliovirus receptor; SNP: Single nucleotide polymorphism; SSC: Side scatter; VPD450: Violet proliferation dye 450

\section{Acknowledgements}

Authors are thankful to Ana Luiza Ongaro Seidinger Conte for preforming Sanger polymorphism screening, and the team of LCE-CAISM for technical support for the karyotyping, and CA125 and HE4 determinations.

\section{Funding}

This study was supported by grants from São Paulo Research Foundation FAPESP. Financial supports numbers 2011/14520-0, 2012/12802-1, 2014/ 14777-1, 2016/07822-4 and scholarship 2014/07401-3. Funding agency did not participate in the design of the study and collection, analysis, and interpretation of data and in writing the manuscript.

\section{Availability of data and materials}

The datasets used and/or analyzed during the current study are available from the corresponding author on reasonable request.

\section{Authors' contributions}

RFS, SFD, JAY and FG analyzed, interpreted the data and wrote the manuscript. RFS, DMC, GOLR, CNSA and NAM performed the experiments, contributing for acquisition of data and their analysis. DMC, GOLR analyzed and interpreted the comparative genomic hybridization data. SFD analyzed and interpreted the patient clinical data. LALAA analyzed and interpreted the patient histopathological data. JAY and FG designed and managed the study. All authors read and approved the final manuscript.

\section{Authors' information}

JAY and SFD are Fellowship from the Brazilian National Counsel of Technological and Scientific Development.

\section{Ethics approval and consent to participate}

The study was approved by the Research Ethics Committee of University of Campinas (27 September 2011, 897/2011) and was registered on the Brazilian National Health Council (CAAE: 0807.0.146.000-11). Patient provided written informed consent for the use of her blood and ascites samples, and her data.

\section{Consent for publication}

A written informed consent for publication of patient's information was provided by the patient's next of kin.

\section{Competing interests}

Sophie Françoise Derchain is an editorial board member of BMC Cancer. The authors declare that they have no competing interests.

\section{Publisher's Note}

Springer Nature remains neutral with regard to jurisdictional claims in published maps and institutional affiliations.

\section{Author details}

${ }^{1}$ Faculdade de Ciências Médicas, University of Campinas, Campinas, SP, Brazil. ${ }^{2}$ Instituto de Biologia, University of Campinas, Campinas, SP, Brazil. ${ }^{3}$ Laboratório de Biologia Molecular, Centro Infantil Boldrini, Campinas, SP, Brazil. "Women's Hospital "Professor Doutor José Aristodemo Pinotti" CAISM, University of Campinas, Rua Alexander Fleming 101, Campinas, SP 13083-881, Brazil.

Received: 26 January 2017 Accepted: 30 October 2017

Published online: 13 November 2017

\section{References}

1. Naora H, Montell DJ. Ovarian cancer metastasis: integrating insights from disparate model organisms. Nat Rev Cancer. 2005;5(5):355-66.

2. Siegel RL, Miller KD, Jemal A. Cancer statistics, 2015. CA Cancer J Clin 2015;65(1):5-29

3. Bowtell DD. The genesis and evolution of high-grade serous ovarian cancer. Nat Rev Cancer. 2011;10(11):803-8.

4. Vaughan S, Coward JI, Bast Jr RC, Berchuck A, Berek JS, Brenton JD, et al. Rethinking ovarian cancer: recommendations for improving outcomes. Nat Rev Cancer. 2011;11:719-25. doi: 10.1038/nrc3144.

5. Vargas AN. Natural history of ovarian cancer. Cancer Science \&Therapy. 2014;6:247-52.

6. Seidman JD, Horkayne-Szakaly I, Haiba M, Boice CR, Kurman RJ, Ronnett BM The histologic type and stage distribution of ovarian carcinomas of surface epithelial origin. Int J Gynecol Pathol. 2004;23(1):41-4.

7. Galgano MT, Hampton GM, Frierson HF Jr. Comprehensive analysis of HE4 expression in normal and malignant human tissues. Mod Pathol. 2006;19(6):847-53. 
8. Pitta DR, Sarian LO, Barreta A, Campos EA, Andrade LL, Fachini AM, et al. Symptoms, CA125 and HE4 for the preoperative prediction of ovarian malignancy in Brazilian women with ovarian masses. BMC Cancer. 2013;13:423. doi: 10.1186/1471-2407-13-423.

9. Häusler SF, Montalbán del Barrio I, Strohschein J, Chandran PA, Engel JB, Hönig A, et al. Ectonucleotidases CD39 and CD73 on OvCA cells are potent adenosine generating enzymes responsible for adenosine receptor 2Adependent suppression of T cell function and NK cell cytotoxicity. Cancer Immunol Immunother. 2011;60(10):1405-18.

10. Patriarca C, Macchi RM, Marschner AK, Mellstedt H. Epithelial cell adhesion molecule expression. (CD326) in cancer: a short review. Cancer Treat Rev. 2012;38:68-75

11. O'Donnell RL, McCormick A, Mukhopadhyay A, Woodhouse LC, Moat M, Grundy A, et al. The use of ovarian cancer cells from patients undergoing surgery to generate primary cultures capable of undergoing functional analysis. PLoS One. 2014;9(6):e90604.

12. Huang RY, Chen GB, Matsumura N, Lai HC, Mori S, Li J, Wong MK, Konishi I, Thiery JP, Goh LBMC. Histotype-specific copy-number alterations in ovarian cancer. Med Genomics. 2012;5:47. doi: 10.1186/1755-8794-5-47.

13. Zhang $\mathrm{D}, \mathrm{Chen} \mathrm{P}$, Zheng $\mathrm{CH}$, Xia J. Identification of recurrent focal copy number variations and their putative targeted driver genes in ovarian cancer. BMC Bioinformatics Oncotarget. 2016;7(4):4298-309.

14. Thériault BL, Portelance L, Mes-Masson AM, Nachtigal MW. Establishment of primary cultures from ovarian tumor tissue and ascites fluid. In: Malek A, Tchernitsa O, editors. Ovarian cancer: methods and protocols, methods in molecular biology. Secaucus: Springer; 2013. p. 323-36.

15. Chen J, Wang J, Zhang Y, Chen D, Yang C, Kai C, Wang X, Shi F, Dou J. Observation of ovarian cancer stem cell behavior and investigation of potential mechanisms of drug resistance in three-dimensional cell culture. J Biosci Bioeng. 2014;118(2):214-22.

16. Giri S, Rattan R, Deshpande M, Maguire JL, Johnson Z, Graham RP, Shridhar $V$. Preclinical therapeutic potential of a nitrosylating agent in the treatment of ovarian cancer. PLoS One. 2014;9(6):e97897.

17. Jacob F, Nixdorf S, Hacker NF, Heinzelmann-Schwarz VA. Reliable in vitro studies require appropriate ovarian cancer cell lines. J Ovarian Res. 2014;7: 60. doi: 10.1186/1757-2215-7-60.

18. Barretina J, Caponigro G, Stransky N, Venkatesan K, Margolin AA, Wilson CJ et al. The cancer cell line encyclopedia enables predictive modeling of anticancer drug sensitivity. Nature. 2012;483(7391):603-7.

19. Domcke S, Sinha R, Levine DA, Sander C, Schults N. Evaluating cell lines as tumor models by comparison of genomic profiles. Nat Commun. 2013;4: 2126-36. doi: 10.1038/ncomms3126.

20. Sugimoto T, Gotoh T, Yagyu S, Kuroda H, lehara T, Hosoi H, Ohta S, Ohira M, Nakagawara A. A MYCN-amplified cell line derived from a long-term event-free survivor among our sixteen established neuroblastoma cell lines. Cancer Lett. 2013:331:115-21.

21. Arcila M, Lau C, Nafa K, Ladanyi M. Detection of KRAS and BRAF mutations in colorectal carcinoma roles for high-sensitivity locked nucleic acid-PCR sequencing and broad-spectrum mass spectrometry genotyping. J Mol Diagn. 2011;13(1):64-73.

22. Kuo KT, Guan B, Feng B, MaoTL CX, Jinawath N, Wang Y, Kurman RJ, Shih IM, Wanf TL. Analysis of DNA copy number alterations in ovarian serous tumors identifies new molecular genetic changes in low-grade and high-grade carcinomas. Cancer Res. 2009;69(9):40364042. doi: 10.1158/0008-5472.CAN-08-3913.

23. Boyd J, Luo B, Peri S, Wirchansky B, Hughes L, Forsythe C, Wu H. Whole exome sequence analysis of serous borderline tumors. Gynecologic Oncol. 2013;130:560-4

24. Hunter SM, Anglesio MS, Ryland GL, et al. Molecular profiling of low grade serous ovarian tumours identifies novel candidate driver genes. Oncotarget. 2015;6:37663-77.

25. Fernández ML, DiMattia GE, Dawson A, Bamford S, Anderson S, Hennessy BT, Anglesio MS, Shepherd TG, Salamanca C, Hoenisch J, Tinker A, Huntsman DG, Carey MS. Differences in MEK inhibitor efficacy in molecularly characterized low-grade serous ovarian cancer cell lines. Am J Cancer. 2016;6:2235-51.

26. Lee JY, Yoon JK, Kim B, Kim S, Kim MA, Lim H, et al. Tumor evolution and intratumor heterogeneity of an epithelial ovarian cancer investigated using nextgeneration sequencing. BMC Cancer. 2015;15:85. doi: 10.1186/s12885-015-1077-4.

27. Freeman JL, Perry GH, Feuk L, Redon R, McCarroll SA, Altshuler DM, et al. Copy number variation: new insights in genome diversity. Genome Res. 2006;16(8):949-61.
28. Emmanuel C, Chiew YE, George J, Etemadmoghadam D, Anglesio MS, Sharma R, Russell P, Kennedy C, Fereday S, Hung J, Galletta L, Hogg R, Wain GV, Brand A, Balleine R, MacConaill L, Palescandolo E, Hunter SM, Campbell I, Dobrovic A, Wong SQ, Do H, Clarke CL, Harnett PR, Bowtell DDL, DeFazio A. Genomic classification of serous ovarian cancer with adjacent borderline differentiates RAS pathway and TP53-mutant tumors and identifies NRAS as an oncogenic driver. Clin Cancer Res. 2014;20:6618-30.

29. Mclntyre JB, Rambau PF, Chan A, Yap S, Morris D, Nelson GS, Köbel M. Molecular alterations in indolent, aggressive and recurrent ovarian lowgrade serous carcinoma. Histopathology. 2017;70:347-58. doi: 10.1111/his.13071.

30. Della Pepa C, Tonini G, Santini D, Losito S, Pisano C, Di Napoli M, Cecere SC, Gargiulo P, Pignata S. Low grade serous ovarian carcinoma: from the molecular characterization to the best therapeutic strategy. Cancer Treat Rev. 2015:41:136-43.

31. Masciullo V, Baldassarre G, Pentimalli F, Berlingieri MT, Boccia A, Chiappetta G, Palazzo J, Manfioletti G, Giancotti V, Viglietto G, Scambia G, Fusco A. HMGA1 protein over-expression is a frequent feature of epithelial ovarian carcinomas. Carcinogenesis. 2003;24:1191-8. doi: 10.1093/carcin/bgg075.

32. Wang M, Wang J, Wang L, Wu L, Xin X. Notch1 expression correlates with tumor differentiation status in ovarian carcinoma. Med Oncol. 2010;27:1329-35. doi: 10.1007/s12032-009-9384-8.

33. Alniaimi AN, Demorest-Hayes K, Alexander VM, Seo S, Yang D, Rose S. Increased Notch1 expression is associated with poor overall survival in patients with ovarian cancer. Int J Gynecol Cancer. 2015;25:208-13. doi: 10.1097/IGC.0000000000000359.

34. Carlsten M, Björkström NK, Norell H, Bryceson $Y$, van Hall T, Baumann BC, et al. DNAX accessory molecule-1 mediated recognition of freshly isolated ovarian carcinoma by resting natural killer cells. Cancer Res. 2007;67(3):1317-25.

35. da Silva RF, Petta CA, Derchain SF, Alici E, Guimarães F. Up-regulation of DNAM-1 and NKp30, associated with improvement of NK cells activation after long-term culture of mononuclear cells from patients with ovarian neoplasia. Hum Immunol. 2014;75(8):777-84

36. Schanoski AS, Cavalcanti TC, Campos CBL, Vieira-Matos AN, Rettori O, Guimarães F. Walker 256 tumor MHC class I expression during the shift a variant to the immunogenic AR variant. Cancer Lett. 2004;211(1):119-27.

37. Hirohashi Y, Torigoe T, Mariya T, Kochin V, Saito T, Sato N. HLA class I as a predictor of clinical prognosis and CTL infiltration as a predictor of chemosensitivity in ovarian cancer. Oncoimmunology. 2015;4(5):e1005507. doi: 10.1080/2162402X.2015.1005507.

38. Ghiringhelli F, Ménard C, Terme M, Flament C, Taieb J, Chaput N, et al. CD4 +CD25+ regulatory $T$ cells inhibit natural killer cell functions in a transforming growth factor-B-dependent manner. J Exp Med. 2005;202(8):1075-85.

39. Young A, Mittal D, Stagg J, Smyth MJ. Targeting cancer-derived adenosine: new therapeutic approaches. Cancer Discov. 2014;4(8):879-88.

\section{Submit your next manuscript to BioMed Central and we will help you at every step:}

- We accept pre-submission inquiries

- Our selector tool helps you to find the most relevant journal

- We provide round the clock customer support

- Convenient online submission

- Thorough peer review

- Inclusion in PubMed and all major indexing services

- Maximum visibility for your research

Submit your manuscript at www.biomedcentral.com/submit 